\title{
Research Article \\ Effect of Pore Size on the Biodegradation Rate of Silk Fibroin Scaffolds
}

\author{
Zuwei Luo, ${ }^{1}$ Qin Zhang, ${ }^{1}$ Meijing Shi, ${ }^{1}$ Yang Zhang, ${ }^{1}$ Wei Tao, ${ }^{2}$ and Mingzhong Li ${ }^{1}$ \\ ${ }^{1}$ National Engineering Laboratory for Modern Silk, College of Textile and Clothing Engineering, Soochow University, \\ No. 199 Ren'ai Road, Industrial Park, Suzhou 215123, China \\ ${ }^{2}$ Suzhou SOHO Biomaterial Technology Co., Ltd., No. 105 Tianshangjiang Road, Wuzhong District, Suzhou 215128, China \\ Correspondence should be addressed to Mingzhong Li; mzli@suda.edu.cn
}

Received 14 October 2014; Accepted 27 December 2014

Academic Editor: Vladimir Tsukruk

Copyright (C) 2015 Zuwei Luo et al. This is an open access article distributed under the Creative Commons Attribution License, which permits unrestricted use, distribution, and reproduction in any medium, provided the original work is properly cited.

Controlling the degradation rate of silk fibroin-based biomaterial is an important capability for the fabrication of silk-based tissue engineering scaffolds. In this study, scaffolds with different pore sizes were prepared by controlling the freezing temperature and the silk fibroin concentration. In vitro degradation results showed that the internal pore walls of the scaffolds with a larger pore size collapsed upon exposure to collagenase IA for times ranging from 6 to 12 days, and the silk scaffolds exhibited a faster rate of weight loss. The morphological and structural features of the silk scaffolds with a smaller pore size maintained structural integrity after incubation in the protease solution for 18 days, and the rate of weight loss was relatively slow. Scaffolds with a smaller pore size or a higher pore density degraded more slowly than scaffolds with a larger pore size or lower pore density. These results demonstrate that the pore size of silk biomaterials is crucial in controlling the degradation rate of tissue engineering scaffolds.

\section{Introduction}

Silk fibroin materials can support cell adhesion, growth, proliferation, and differentiation for a large variety of cell types $[1,2]$ and have aroused increasing interest in the field of biomedicine because of their excellent environmental stability, biocompatibility, morphologic flexibility, and mechanical properties [1,3-7]. Silk fibroin materials can be degraded in vitro and in vivo $[3,6,8-13]$, and the degradation products are abundant peptides and some free amino acids, which can be easily metabolized and cleared from the body $[6,10$, $13,14]$. For the tissue engineering scaffolds, one of the key requirements is that the degradation rate should match the rate of new tissue regeneration $[10,15]$. Therefore, controlling the degradation rate of silk fibroin-based materials is an important subject.

As a protein, silk fibroin can be catalytically degraded by proteolytic enzymes. The rate and extent of degradation may be highly variable, depending on a collection of chemical, physical, and biological factors related to the molecularweight distribution $[6,16,17]$ and secondary structure $[9$, $18-20]$ of the silk fibroin, processing methods [15, 21-24], and features of the enzymes [4-6, 12, 25]. Compared with natural silk fibre, regenerated silk fibroin was degraded at a significantly increasing rate in the actinomycetes enzyme solution, because a portion of the natural silk fibroin was hydrolyzed when it was dissolved in neutral salt solution ( $\mathrm{LiBr} \cdot \mathrm{H}_{2} \mathrm{O} \cdot \mathrm{CH}_{3} \mathrm{CH}_{2} \mathrm{OH}$ ), leading to an obvious decrease in the molecular weight and a lower mechanical property during the process of regeneration [26]. Numerous studies in the literature have reported that silk fibroin materials with the lowest $\beta$-sheet content achieved the highest degradation rate $[19,21]$. Three-dimensional porous scaffolds prepared from regenerated silk fibroin using either an all-aqueous process or a process involving an organic solvent, hexafluoroisopropanol (HFIP), were implanted subcutaneously into rats. Most scaffolds prepared by the all-aqueous process degraded to completion between 2 and 6 months, while scaffolds prepared by the organic solvent (HFIP) process persisted beyond 1 year [8]. The degradation behavior of porous silk fibroin materials incubated in $\alpha$-chymotrypsin, collagenase IA, and protease XIV solution demonstrated that $70 \%$ of the sample immersed in protease XIV solution was degraded within 15 days at $37^{\circ} \mathrm{C}$, but the weight loss of the samples 
exposed to collagenase IA and $\alpha$-chymotrypsin was $52 \%$ and $32 \%$, respectively [12]. However, there are few reports regarding the effect of the pore size on the biodegradation behavior of silk fibroin scaffolds. In this study, a system of in vitro degradation experiments with collagenase IA dissolved in PBS was adopted to investigate the relationship between pore size and the degradation rate of silk fibroin scaffolds.

\section{Materials and Methods}

2.1. Preparation of Silk Fibroin Aqueous Solution. Silk fibroin solution was prepared following the procedure described previously $[27,28]$. Briefly, raw Bombyx mori silk consists of two types of proteins, fibroin and sericin. To remove the sericin, the silk was treated three times with 0.05 (wt\%) $\mathrm{Na}_{2} \mathrm{CO}_{3}$ for $30 \mathrm{~min}$ at $98 \sim 100^{\circ} \mathrm{C}$, rinsed thoroughly, and dried at $60^{\circ} \mathrm{C}$ in an oven. The extracted silk fibroin was dissolved in a ternary solvent of $\mathrm{CaCl}_{2} / \mathrm{CH}_{3} \mathrm{CH}_{2} \mathrm{OH} / \mathrm{H}_{2} \mathrm{O}$ (molar ratio $1: 2: 8$ ) at $72 \pm 2^{\circ} \mathrm{C}$ for 1 hour with stirring. The cooled solution was dialyzed in a cellulose membrane tube (MWCO, 9000) against deionized water for 4 days to obtain the regenerated Bombyx mori silk fibroin solution with a concentration of approximately $3.0 \mathrm{wt} \%$. Then, the silk fibroin aqueous solution was concentrated at room temperature under the environment of the flowing air in the fume hood with stirring to obtain a $10.0 \mathrm{wt} \%$ solution.

2.2. Preparation of Silk Fibroin Scaffolds. Four groups of porous silk fibroin scaffolds were prepared as follows. The concentration of silk fibroin solution was diluted to $1.0 \mathrm{wt} \%$ with deionized water, and an appropriate cross-linking agent (polyethylene glycol diglycidyl ether) was added to the solution to achieve a $40 \%$ weight ratio against the total weight of silk fibroin and stirred slowly for $2 \mathrm{~h}$ at $70^{\circ} \mathrm{C}$. After vacuum defoaming, the mixed solution was poured into a stainless steel vessel to make a $2 \mathrm{~mm}$ thick solution and then frozen at $-40^{\circ} \mathrm{C}$ for $6 \mathrm{~h}$. Subsequently, it was lyophilized using a Virtis Genesis 25-LE Freeze Dryer for $48 \mathrm{~h}$ to obtain the scaffolds of group A. The $3.0 \mathrm{wt} \%$ and $10.0 \mathrm{wt} \%$ silk fibroin solutions were treated with the same methods as group A to prepare the scaffolds of group B and group C, respectively. After the same treatment as group $\mathrm{A}$, a sample of $10.0 \mathrm{wt} \%$ silk fibroin was frozen at $-80^{\circ} \mathrm{C}$ for $6 \mathrm{~h}$ and then freeze-dried for $48 \mathrm{~h}$ to obtain the scaffolds of group D.

2.3. Observation of the Pore Structure of the Three-Dimensional Scaffolds. The cross-sectional morphology of the porous silk scaffolds was observed with a Hitachi S-4800 scanning electron microscope (SEM, Japan) [28]. The SEM images were processed by computer in bmp form. The border of each pore in the top layer was defined according to a gradient method and the top layer pictures of the cross sections were obtained. Each pore area $\left(s_{1}, s_{2}, \ldots, s_{i}, \ldots, s_{n}\right)$ and the total area $S\left(\mathrm{~cm}^{2}\right)$ of porous three-dimensional materials within statistical range were calculated from the area of each pore and the number of pixels in the whole picture. The pore diameter $d_{i}(\mu \mathrm{m})$ of each pore, average pore size $\bar{d}(\mu \mathrm{m})$, and pore density $D\left(\mathrm{~cm}^{-2}\right)$ are given by

$$
\begin{gathered}
d_{i}=\sqrt{\frac{4 s_{i}}{\pi}}, \\
\bar{d}=\frac{\sum_{i=1}^{n} d_{i}}{n}, \\
D=\frac{n}{S} .
\end{gathered}
$$

2.4. In Vitro Degradation of Porous Silk Scaffolds. Silk fibroin scaffold from each group was cut into $3 \mathrm{~cm} \times 3 \mathrm{~cm}$ squares and then immersed in $0.05 \mathrm{M}$ PBS ( $\mathrm{pH} \mathrm{7.0)}$ at $37^{\circ} \mathrm{C}$ for $48 \mathrm{~h}$ to eliminate non-cross-linked silk fibroin. A portion of the samples was dried at $105^{\circ} \mathrm{C}$ to calculate the weight of the samples as the initial mass. Each fibroin square was incubated in $1.0 \mathrm{UmL}^{-1}$ collagenase IA (Sigma, USA) dissolved in 0.05 M PBS ( $\mathrm{pH} 7.0$ ) at $37^{\circ} \mathrm{C}$. After 1, 3, 6, 12, and 18 days, at least three of the fibroin squares were removed, and the enzyme containing the degradation products was replaced with freshly prepared enzyme solution. Samples were immersed in $0.05 \mathrm{M}$ PBS without enzyme as controls.

2.5. Scanning Electron Microscopy (SEM). The residual substances of the porous silk scaffolds degraded by collagenase IA for different lengths of time were collected and lyophilized. The surface morphologies of the scaffolds were imaged with a Hitachi S-4800 scanning electron microscope after sputtering with platinum.

2.6. Weight Change of Porous Silk Scaffolds during Degradation. After 1,3,6,12, and 18 days, samples were collected from each group and first rinsed with deionized water and then lyophilized to calculate the percentage of weight retention.

\section{Results}

3.1. Pore Size of Porous Silk Scaffolds. The four groups of silk fibroin scaffolds showed a significant difference in pore morphology, as shown in Figure 1. Compared with the other three groups of porous silk scaffolds, the scaffolds of group A, prepared from a low concentration of silk fibroin solution, possessed a larger pore size and a thinner pore wall but a smaller pore density. The pore size of group B scaffolds was larger than group $\mathrm{C}$ and $\mathrm{D}$ scaffolds but smaller than that of group A scaffolds. The pore densities of group A and B scaffolds were obviously smaller compared to group $\mathrm{C}$ and D scaffolds. Group D scaffolds exhibited a smaller pore size, a thicker pore wall, and a higher pore density due to the high silk fibroin concentration and low freezing temperature. As described in Figure 2, with an increase in silk fibroin concentration, the average pore size was smaller, but the pore density was larger. The concentrations of group $\mathrm{C}$ and group D silk fibroin solution were both $10.0 \mathrm{wt} \%$; however, the average pore size of group $\mathrm{C}$ scaffolds frozen at $-40^{\circ} \mathrm{C}$ was remarkably larger than that of group $\mathrm{D}$ scaffolds frozen at $-80^{\circ} \mathrm{C}$, while the pore density of group $\mathrm{C}$ scaffolds was smaller. 


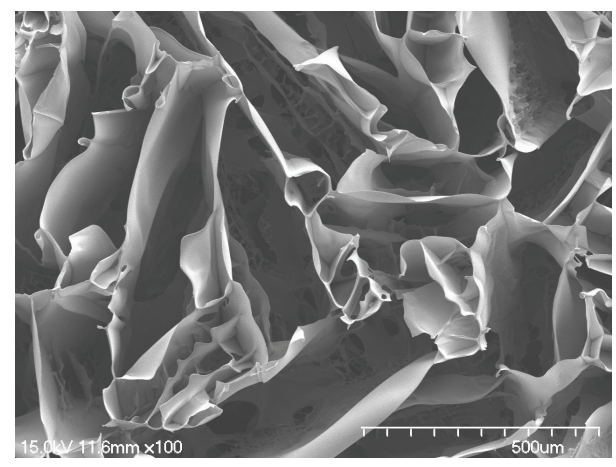

A

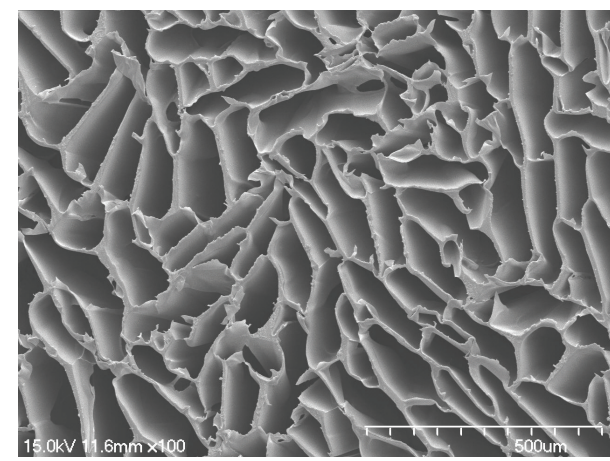

C

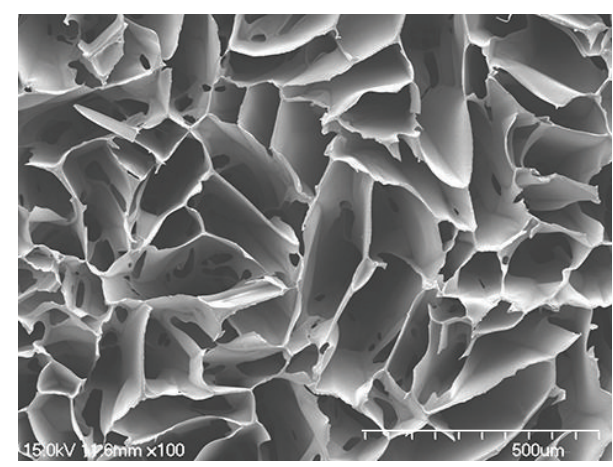

B

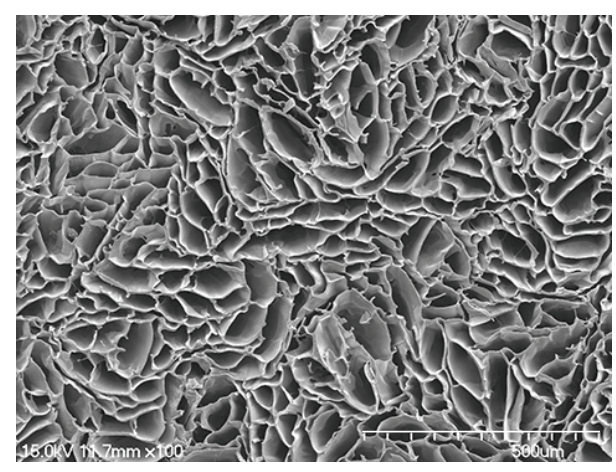

$\mathrm{D}$

FIGURE 1: SEM images of different scaffolds' cross sections (scale bar: $500 \mu \mathrm{m}$ ).

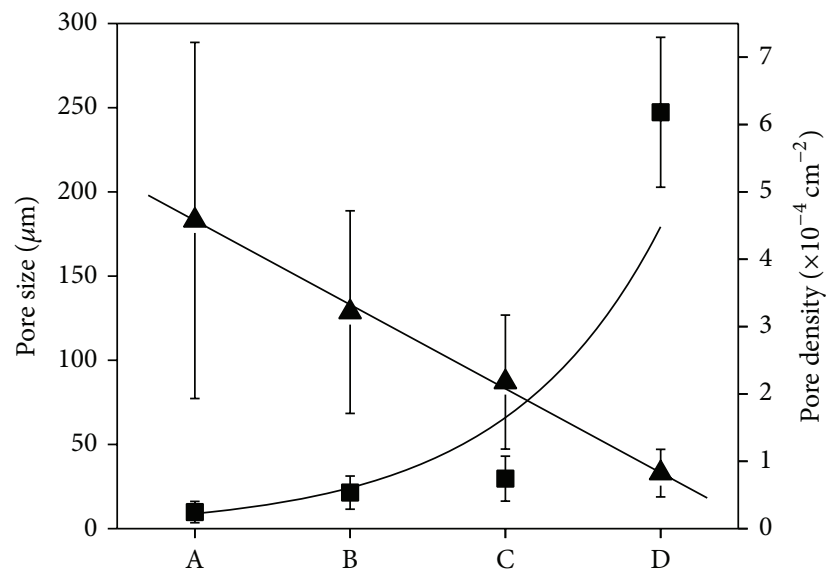

A Pore size

- Pore density

Figure 2: Pore size and pore density of different scaffolds.

3.2. Morphological Changes of Different Porous Silk Scaffolds during Degradation. It can be observed from Figure 3 that there was no remarkable difference among the four groups of scaffolds after they were immersed in collagenase IA solution for three days. However, with increasing incubation time in collagenase IA solution, significant differences in the shape and dimension of porous silk scaffolds with different pore sizes had taken place. After exposure to collagenase IA for times ranging from 6 to 12 days, group A and B fibroin

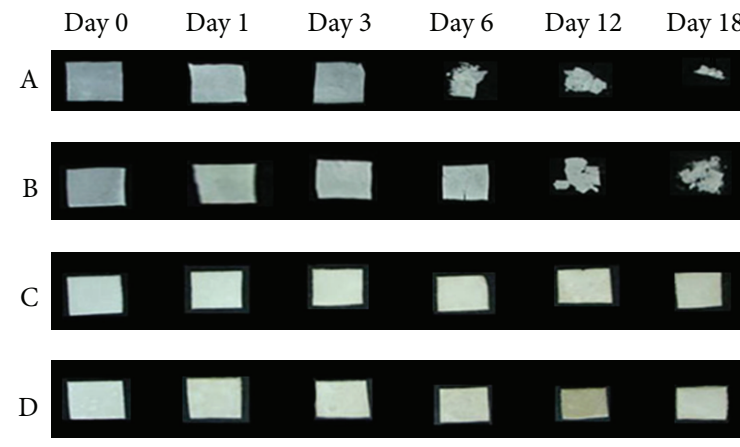

FIGURE 3: Images of the four groups of porous silk scaffolds after degradation by collagenase IA $\left(1.0 \mathrm{UmL}^{-1}, \mathrm{pH} 7.4,37^{\circ} \mathrm{C}\right)$ for different numbers of days.

scaffolds could not retain their original shape, respectively, and broke into several pieces. Furthermore, the remaining fragments decreased dramatically as the degradation time increased. Comparatively, the morphologies of group $\mathrm{C}$ and D scaffolds, both of which possessed smaller pore sizes, remained intact, but the dimensions shrunk remarkably after incubation in collagenase IA solution for 18 days. The observations of the materials' morphologies described in Figure 3 revealed that the pore size of the scaffold had an evident impact on its degradation rate, and the scaffolds with a larger pore size degraded more rapidly.

Figure 4 depicts SEM images of the four groups of porous silk scaffolds incubated in collagenase IA at specific biodegradation time points (6th and 18th days), respectively. 
It can be observed that a series of marked cross-sectional morphological changes have occurred and there were obvious differences among the four groups of scaffolds. After the 6 th day, scaffolds of both group A and group B with larger pore sizes could not retain the original morphologies, with most pores having collapsed and some cracks appearing on the surface of the images. In contrast, group $\mathrm{C}$ and $\mathrm{D}$ scaffolds with a smaller pore size and a higher pore density were still structurally intact, exhibiting little collapse. The differences become more obvious up to 18 days, when all the pores of group A and B scaffolds had corroded into sloppy bulks and powder. By contrast, group C scaffolds demonstrated no evident collapse, showing a little crack, and the pore structure remained regular in group D scaffolds. The results illustrated in Figure 4 indicate that scaffolds possessing a larger pore size and a smaller pore density underwent a greater extent of degradation.

3.3. Weight Changes during Degradation. The weight changes of the four groups of scaffolds with different pore sizes after incubation in collagenase IA for 18 days were shown in Figure 5. With the increasing enzymatic degradation time, the residual weight of the four groups of scaffolds decreased gradually, indicating that the protease-catalyzed degradation resulted in a decrease in weight of the silk fibroin scaffolds. At each time point, there were marked differences among the four groups of scaffolds. After 1 day, scaffolds of both group $A$ and group B underwent a higher level of weight loss, and the residual weight of group A and group B materials was $82.9 \%$ and $92.5 \%$, respectively. The weight losses of group C and $\mathrm{D}$ scaffolds were negligible, not exceeding $3.0 \%$ of the initial weights. Following further degradation, the difference of residual weight among group A, B, C, and D scaffolds significantly increased, yielding residual weight of $71.8 \%$ in group A and $82.8 \%$ in group B at 6 days, while the weights of group $\mathrm{C}$ and $\mathrm{D}$ scaffolds were only reduced by approximately 4.6\%. After degradation for 18 days, the residual weight of group A and B materials was $42.1 \%$ and $49.6 \%$, respectively. Group C scaffold exhibited $83.7 \%$ weight retention, and the residual weight of group D was up to $91.3 \%$. The results demonstrated that the degradation rates of the four groups of scaffolds with various pore sizes and pore densities were obviously different. Group A and B scaffolds showed a more rapid degradation rate due to their larger pore sizes and smaller pore densities than group C and D scaffolds with smaller pore sizes and higher pore densities.

\section{Discussion}

Freeze-drying is commonly employed to prepare porous polymer scaffolds, such as from silk fibroin and collagen $[28,29]$, and a variety of materials with different pore sizes can be fabricated by controlling the freezing temperature and the polymer concentration [30]. In this research, we prepared porous silk scaffolds with varying pore sizes by adjusting the freezing temperature and the fibroin concentration. As shown in Figures 1 and 2, group A and B porous scaffolds exhibit larger pore sizes and smaller pore densities, as well as thinner pore walls due to their low silk fibroin concentration. When group A and B silk solutions were frozen, larger ice particles could be formed inside the silk solution, decreasing in the number of ice nuclei. When the ice crystals were sublimated by vacuum drying, the pores formed in the two groups of porous silk materials were larger than in group C, but the pore densities were smaller than in group C. Keeping the silk fibroin solution at $-80^{\circ} \mathrm{C}$ for quick freezing can cause fibroin temperature to rapidly cross the glass transition temperature region. It was difficult to form larger particles because of the short growth time of the ice crystals. Therefore, when the ice crystals were sublimated by vacuum drying, the pore size in group $\mathrm{D}$ material frozen at $-80^{\circ} \mathrm{C}$ was smaller than the pore size of group $\mathrm{C}$ material, which shared the same concentration but was frozen at $-40^{\circ} \mathrm{C}$.

Arai et al. [3] have studied the in vitro biodegradation of Bombyx mori silk fibroin by incubating fibres and films with proteolytic enzymes (collagenase type F, $\alpha$-chymotrypsin type I-S, and protease type XXI), for times ranging from 1 to 17 days. The results indicated that the changes in sample weight and degree of polymerisation of silk fibres exposed to proteolytic attack were negligible, but the tensile properties were significantly reduced. The silk films exhibited a noticeable decrease in sample weight and mechanical properties. In Horan's study [10], Bombyx mori silk fibroin yarns were incubated in protease XIV to create an in vitro model system of proteolytic degradation and harvested at designated time points up to 12 weeks. The silk fibroin demonstrated a decline in fibroin diameter and strength with time of exposure to the enzyme. These results suggested that silk fibroin is biodegradable, and the degradation mechanism is the enzyme-catalyzed hydrolysis. The characteristics of silk biodegradation behaviors varied with different types of enzymes [14].

In this study, the in vitro degradation behavior of the four groups of porous silk fibroin scaffolds with different pore sizes prepared by freeze drying was investigated. Upon exposure to collagenase IA for various times, significant changes occurred in the morphologies of the four groups of scaffolds, as shown in Figures 3 and 4. As shown in Figure 5, remarkable differences in weight loss among the four groups of scaffolds can be observed during the process of degradation. The degradation rate and extent of the four groups of scaffolds were in the order A > B > C > D. Much more protease solution could be contained by per unit volume of scaffolds with larger pore sizes and high porosities (Figures $1(\mathrm{~A})$ and $1(\mathrm{~B})$ ), providing a high possibility for the pore walls to contact the protease molecules. Consequently, the scaffolds had more opportunities to be degraded by protease. Meanwhile, the porous structures of the scaffolds were supported by pore walls. Larger pore sizes led to a decrease in the amount of pore walls per unit volume of scaffolds (Figures $1(\mathrm{~A})$ and 1(B)). The degradation of a portion of pore walls by proteasecatalyzed hydrolysis resulted in a further decrease in the number of the residual pore walls playing a crucial role in supporting the porous structures of the scaffolds. Therefore, the scaffolds were easy to collapse (Figures 4(A) and 4(B). Group A and B fibroin materials with larger pore sizes created favorable conditions for protease molecule to fully contact 


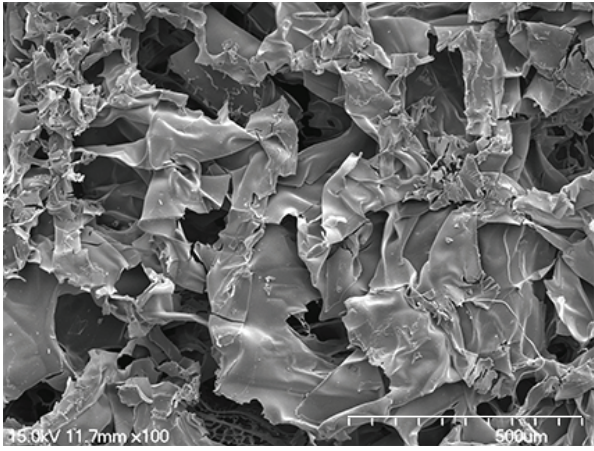

$\mathrm{A}_{6}$

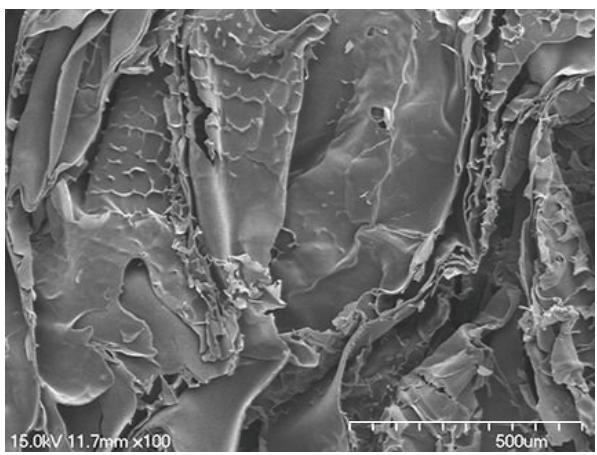

$\mathrm{B}_{6}$

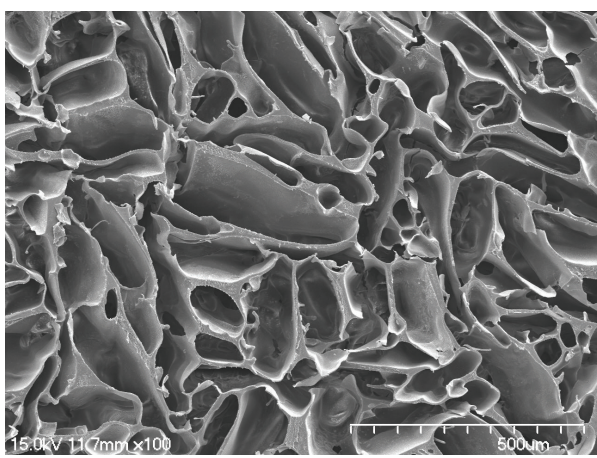

$\mathrm{C}_{6}$

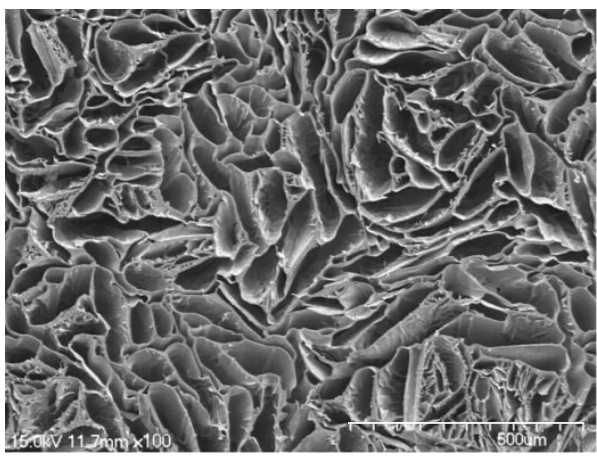

$\mathrm{D}_{6}$

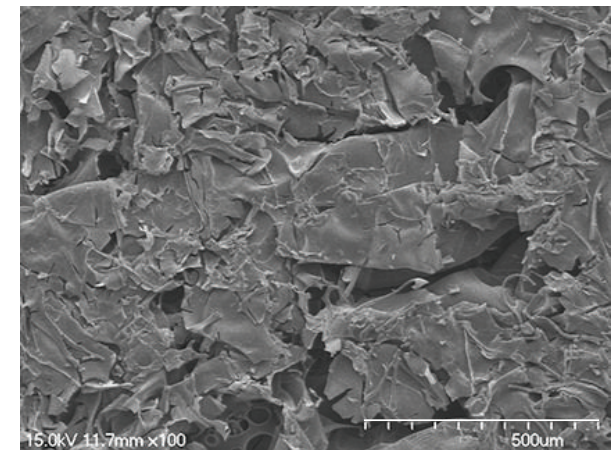

$\mathrm{A}_{18}$

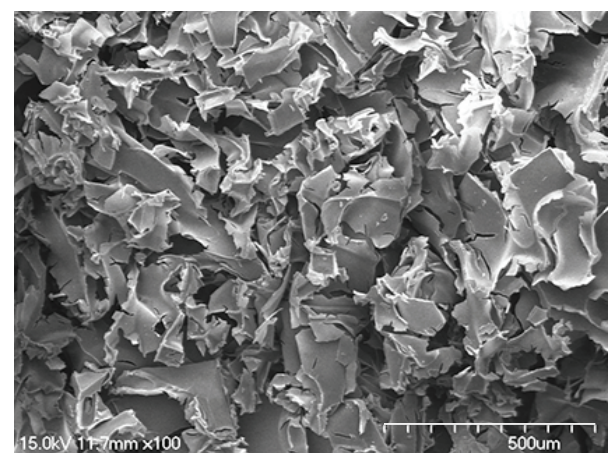

$\mathrm{B}_{18}$

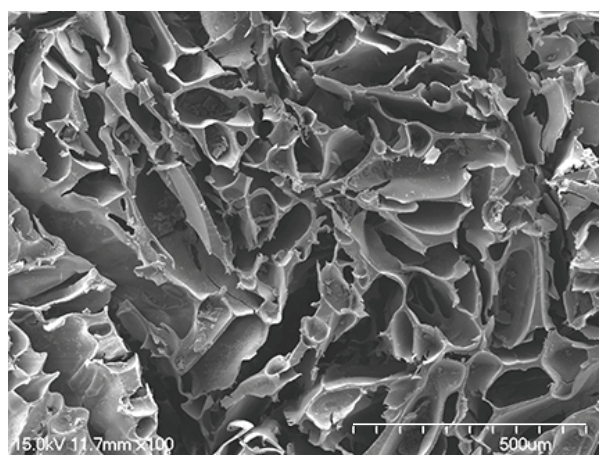

$\mathrm{C}_{18}$

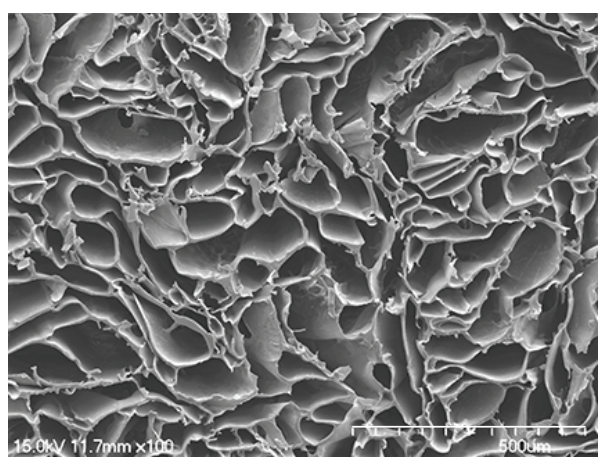

$\mathrm{D}_{18}$

Figure 4: SEM images of four groups of porous silk fibroin scaffolds after degradation by collagenase IA for 6 days $\left(\mathrm{A}_{6}, \mathrm{~B}_{6}, \mathrm{C}_{6}\right.$, and $\left.\mathrm{D}_{6}\right)$ and 18 days $\left(\mathrm{A}_{18}, \mathrm{~B}_{18}, \mathrm{C}_{18}\right.$, and $\left.\mathrm{D}_{18}\right)$ (scale bar: $\left.500 \mu \mathrm{m}\right)$. 


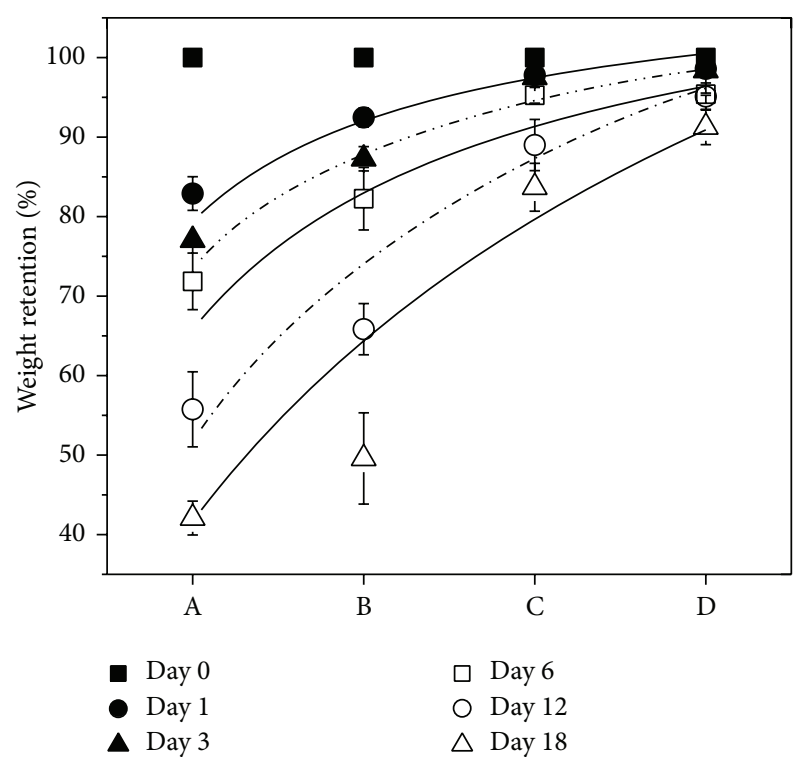

FIgURE 5: Weight change of the four groups of porous silk fibroin scaffolds during enzymatic degradation.

the pore walls and then penetrate into the interior of the walls, which consequently resulted in higher degradation rates compared to group $\mathrm{C}$ and $\mathrm{D}$ materials with smaller pore sizes.

\section{Conclusions}

Silk fibroin scaffolds with various pore sizes were prepared by controlling the freezing temperature and the concentration of silk fibroin. The in vitro degradation results of fibroin scaffolds incubated in collagenase IA suggested that the internal pore walls of the scaffolds with a larger pore size collapsed upon exposure to protease for times ranging from 6 to 12 days, and these silk scaffolds exhibited a faster rate of weight loss. The morphological and structural features of the silk scaffolds with smaller pore size remained intact after incubation in the protease solution for 18 days, and the rate of weight loss was relatively slow. Scaffolds with a smaller pore size or a higher pore density degraded more slowly than scaffolds with a larger pore size or a lower pore density.

\section{Conflict of Interests}

The authors declare that there is no conflict of interests regarding the publication of this paper.

\section{Acknowledgments}

This work was supported by the National Nature Science Foundation of China (31370968), Natural Science Foundation of Jiangsu Province (BK20131177), College of Natural Science Research Project of Jiangsu Province (12KJA430003), and Jiangsu Province Science and Technology Support Program (BE2013734).

\section{References}

[1] O. J. Lee, J. M. Lee, J. H. Kim, H. Kweon, Y. Y. Jo, and C. H. Park, "Biodegradation behavior of silk fibroin membranes in repairing tympanic membrane perforations," Journal of Biomedical Materials Research Part A, vol. 100, no. 8, pp. 20182026, 2012.

[2] C. X. Zhao, X. F. Wu, Q. Zhang, S. Q. Yan, and M. Z. $\mathrm{Li}$, "Enzymatic degradation of Antheraea pernyi silk fibroin 3D scaffolds and fibers," International Journal of Biological Macromolecules, vol. 48, no. 2, pp. 249-255, 2011.

[3] T. Arai, G. Freddi, R. Innocenti, and M. Tsukada, "Biodegradation of Bombyx mori silk fibroin fibers and films," Journal of Applied Polymer Science, vol. 91, no. 4, pp. 2383-2390, 2004.

[4] N. Sasithorn and L. Martinová, "Fabrication of silk nanofibres with needle and roller electrospinning methods," Journal of Nanomaterials, vol. 2014, Article ID 947315, 9 pages, 2014.

[5] Y. Srisuwan and Y. Baimark, "Preparation of biodegradable silk fibroin/alginate blend films for controlled release of antimicrobial drugs," Advances in Materials Science and Engineering, vol. 2013, Article ID 412458, 6 pages, 2013.

[6] Y. Cao and B. Wang, "Biodegradation of silk biomaterials," International Journal of Molecular Sciences, vol. 10, no. 4, pp. 1514-1524, 2009.

[7] E. S. Gil, S.-H. Park, X. Hu, P. Cebe, and D. L. Kaplan, "Impact of sterilization on the enzymatic degradation and mechanical properties of silk biomaterials," Macromolecular Bioscience, vol. 14, no. 2, pp. 257-269, 2014.

[8] Y. Wang, D. D. Rudym, A. Walsh et al., "In vivo degradation of three-dimensional silk fibroin scaffolds," Biomaterials, vol. 29, no. 24, pp. 3415-3428, 2008.

[9] Q. Lu, B. Zhang, M. Z. Li et al., "Degradation mechanism and control of silk fibroin," Biomacromolecules, vol. 12, no. 4, pp. 1080-1086, 2011.

[10] R. L. Horan, K. Antle, A. L. Collette et al., "In vitro degradation of silk fibroin,” Biomaterials, vol. 26, no. 17, pp. 3385-3393, 2005.

[11] Y. M. Yang, Y. H. Zhao, Y. Gu et al., "Degradation behaviors of nerve guidance conduits made up of silk fibroin in vitro and in vivo," Polymer Degradation and Stability, vol. 94, no. 12, pp. 2213-2220, 2009.

[12] M. Li, M. Ogiso, and N. Minoura, "Enzymatic degradation behavior of porous silk fibroin sheets," Biomaterials, vol. 24, no. 2, pp. 357-365, 2003.

[13] J. Zhou, C. Cao, X. Ma, L. Hu, L. Chen, and C. Wang, "In vitro and in vivo degradation behavior of aqueous-derived electrospun silk fibroin scaffolds," Polymer Degradation and Stability, vol. 95, no. 9, pp. 1679-1685, 2010.

[14] Y. Xu, Y. Wang, Y. Jiao, C. Zhang, and M. Li, "Enzymatic degradation properties of silk fibroin film," Journal of Fiber Bioengineering \& Informatics, vol. 4, no. 1, pp. 35-41, 2011.

[15] J. H. Kim, C. H. Park, O.-J. Lee et al., "Preparation and in vivo degradation of controlled biodegradability of electrospun silk fibroin nanofiber mats," Journal of Biomedical Materials Research Part A, vol. 100, no. 12, pp. 3287-3295, 2012.

[16] K. Zhang, A. Yin, C. Huang et al., "Degradation of electrospun SF/P(LLA-CL) blended nanofibrous scaffolds in vitro," Polymer Degradation and Stability, vol. 96, no. 12, pp. 2266-2275, 2011.

[17] E. M. Pritchard, X. Hu, V. Finley, C. K. Kuo, and D. L. Kaplan, "Effect of silk protein processing on drug delivery from silk films," Macromolecular Bioscience, vol. 13, no. 3, pp. 311-320, 2013. 
[18] M. A. Koperska, D. Pawcenis, J. Bagniuk et al., "Degradation markers of fibroin in silk through infrared spectroscopy," Polymer Degradation and Stability, vol. 105, pp. 185-196, 2014.

[19] Y. P. Hu, Q. Zhang, R. C. You, L. S. Wang, and M. Z. Li, “The relationship between secondary structure and biodegradation behavior of silk fibroin scaffolds," Advances in Materials Science and Engineering, vol. 2012, Article ID 185905, 5 pages, 2012.

[20] R. Rajkhowa, X. Hu, T. Tsuzuki, D. L. Kaplan, and X. Wang, "Structure and biodegradation mechanism of milled Bombyx mori silk particles," Biomacromolecules, vol. 13, no. 8, pp. 25032512, 2012.

[21] X. F. Li, C. Zhang, L. S. Wang, C. L. Ma, W. C. Yang, and M. Z. Li, "Acylation modification of Antheraea pernyi silk fibroin using succinic anhydride and its effects on enzymatic degradation behavior," Journal of Chemistry, vol. 2013, Article ID 640913, 7 pages, 2013.

[22] E. M. Pritchard, T. Valentin, D. Boison, and D. L. Kaplan, "Incorporation of proteinase inhibitors into silk-based delivery devices for enhanced control of degradation and drug release," Biomaterials, vol. 32, no. 3, pp. 909-918, 2011.

[23] P. Wongpanit, Y. Tabata, and R. Rujiravanit, "Miscibility and biodegradability of silk fibroin/carboxymethyl chitin blend films," Macromolecular Bioscience, vol. 7, no. 12, pp. 1258-1271, 2007.

[24] H.-Y. Cheung, K.-T. Lau, Y.-F. Pow, Y.-Q. Zhao, and D. Hui, "Biodegradation of a silkworm silk/PLA composite," Composites Part B: Engineering, vol. 41, no. 3, pp. 223-228, 2010.

[25] C. Wongnarat and P. Srihanam, "Degradation behaviors of thai Bombyx mori silk fibroins exposure to protease enzymes ," Engineering, vol. 5, no. 1, pp. 61-66, 2013.

[26] B. Zuo, L. Dai, and Z. Wu, "Analysis of structure and properties of biodegradable regenerated silk fibroin fibers," Journal of Materials Science, vol. 41, no. 11, pp. 3357-3361, 2006.

[27] Q. Zhang, M. Li, W. Xu, J. Li, and S. Yan, "A novel silk fibroin scaffolds with oriented multichannels," Materials Letters, vol. 105, pp. 8-11, 2013.

[28] M. Z. Li, S. Z. Lu, Z. Y. Wu, H. J. Yan, J. Y. Mo, and L. H. Wang, "Study on porous silk fibroin materials. I. Fine structure of freeze dried silk fibroin," Journal of Applied Polymer Science, vol. 79, no. 12, pp. 2185-2191, 2001.

[29] F. J. O'Brien, B. A. Harley, I. V. Yannas, and L. Gibson, "Influence of freezing rate on pore structure in freeze-dried collagen-GAG scaffolds," Biomaterials, vol. 25, no. 6, pp. 1077-1086, 2004.

[30] Y. Z. Du, X. F. Chen, Y. H. Koh, and B. Lei, "Facilely fabricating PCL nanofibrous scaffolds with hierarchical pore structure for tissue engineering," Materials Letters, vol. 122, pp. 62-65, 2014. 

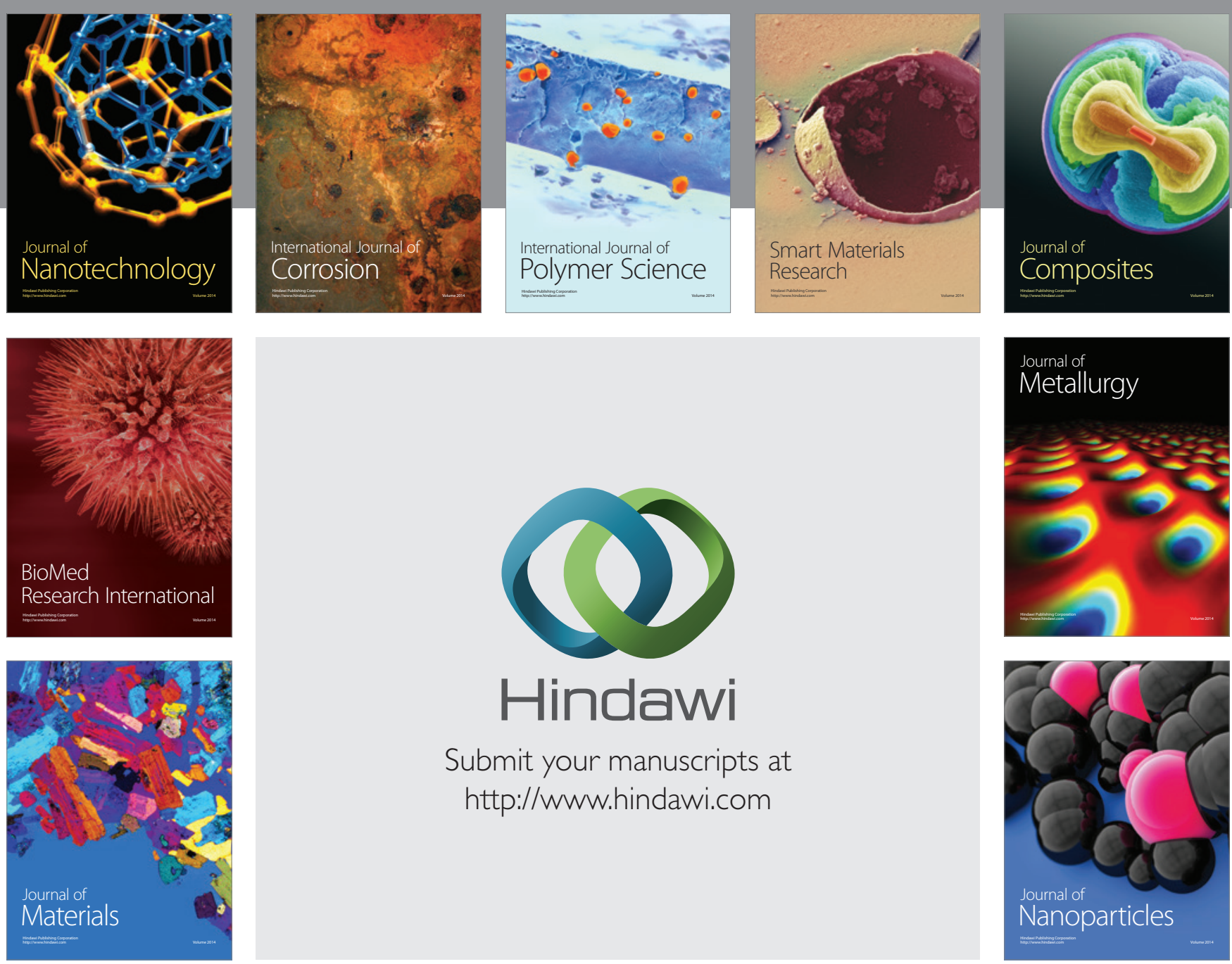

Submit your manuscripts at http://www.hindawi.com
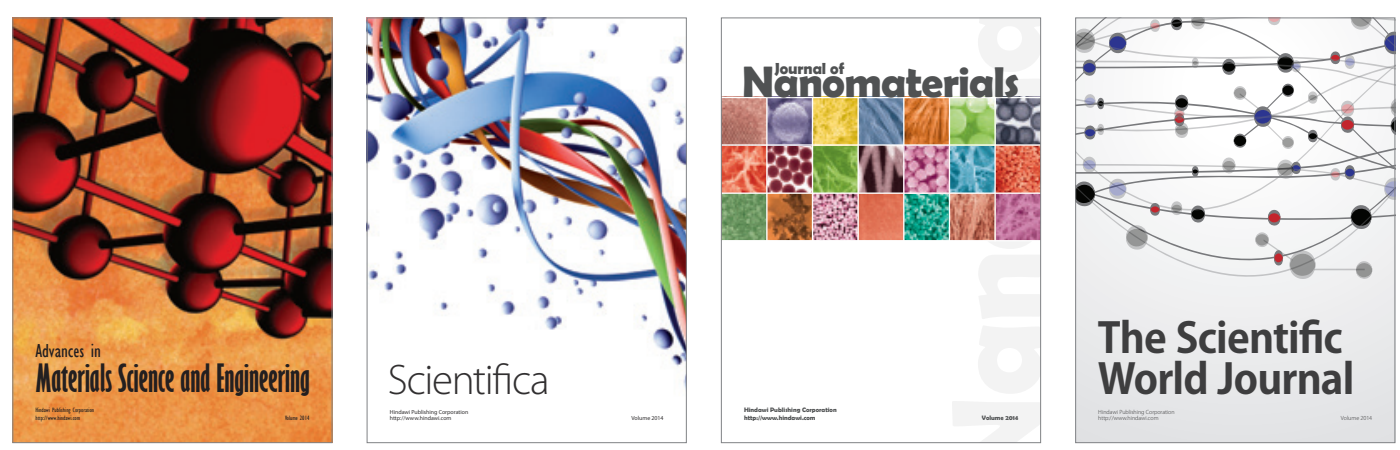

\section{The Scientific World Journal}
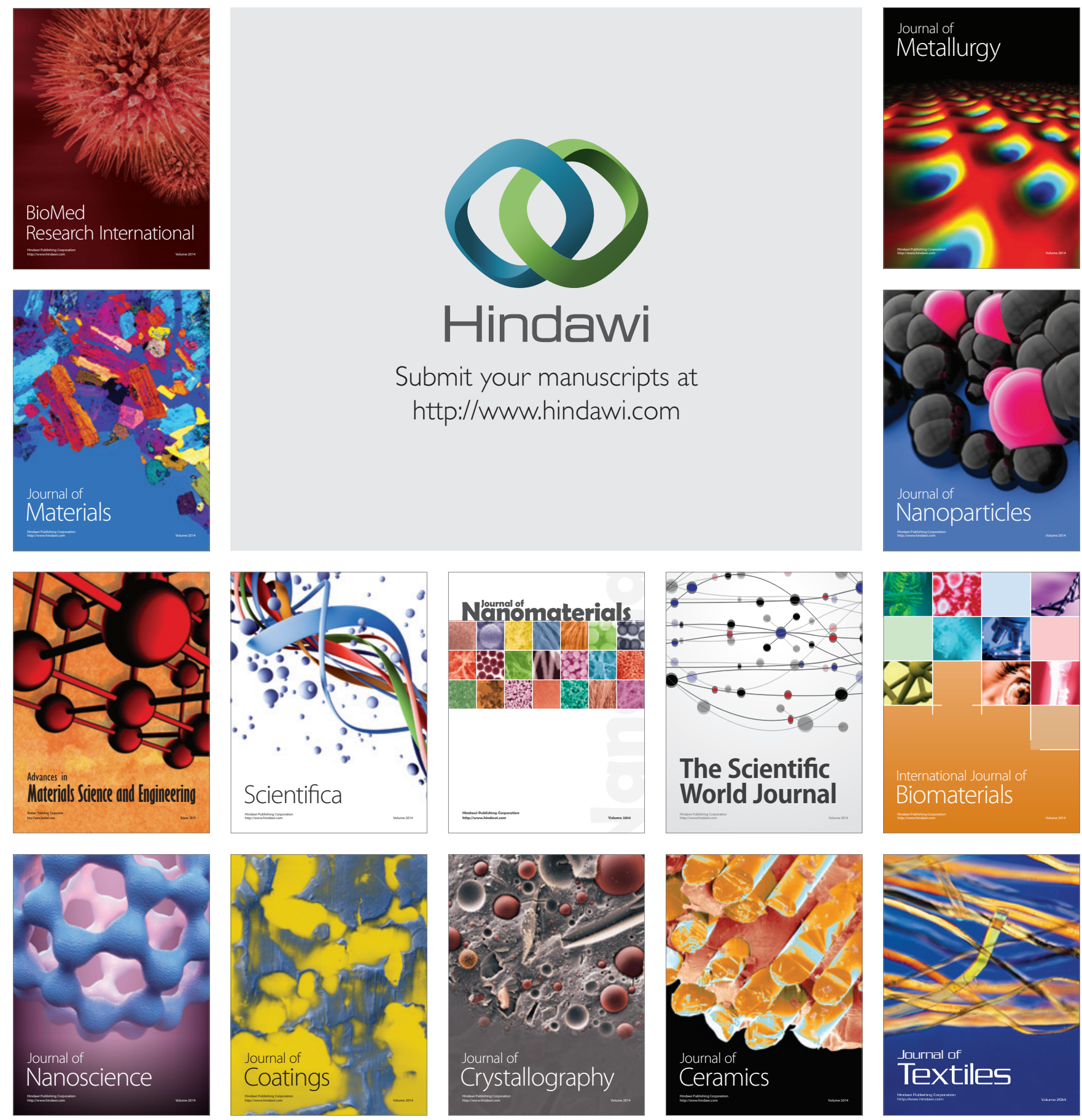